\title{
BMJ Open Dietary fibre to reduce colon cancer risk in Alaska Native people: the Alaska FIRST randomised clinical trial protocol
}

\author{
Kathryn R Koller (D) , , Annette Wilson (i) , ${ }^{2}$ Daniel P Normolle, ${ }^{3}$ \\ Jeremy K Nicholson, ${ }^{4}$ Jia V Li, ${ }^{5}$ James Kinross, ${ }^{6}$ Flora R Lee, ${ }^{1}$ Christie A Flanagan, ${ }^{7}$ \\ Zoe T Merculieff, ${ }^{8}$ Priya Iyer, ${ }^{3}$ Daniela L Lammers, ${ }^{1}$ Timothy K Thomas, ${ }^{1}$ \\ Stephen J D O'Keefe ${ }^{2}$
}

To cite: Koller KR, Wilson A, Normolle DP, et al. Dietary fibre to reduce colon cancer risk in Alaska Native people: the Alaska FIRST randomised clinical trial protocol. BMJ Open 2021;11:e047162. doi:10.1136/ bmjopen-2020-047162

- Prepublication history for this paper is available online To view these files, please visit the journal online (http://dx.doi org/10.1136/bmjopen-2020047162).

Received 21 November 2020 Accepted 09 August 2021

Check for updates

(C) Author(s) (or their employer(s)) 2021. Re-use permitted under CC BY-NC. No commercial re-use. See rights and permissions. Published by BMJ.

For numbered affiliations see end of article.

Correspondence to Dr Kathryn R Koller; kkoller@anthc.org

\section{ABSTRACT}

Introduction Diet, shown to impact colorectal cancer (CRC) risk, is a modifiable environmental factor. Fibre foods fermented by gut microbiota produce metabolites that not only provide food for the colonic epithelium but also exert regulatory effects on colonic mucosal inflammation and proliferation. We describe methods used in a double-blinded, randomised, controlled trial with Alaska Native (AN) people to determine if dietary fibre supplementation can substantially reduce CRC risk among people with the highest reported CRC incidence worldwide.

Methods and analyses Eligible patients undergoing routine screening colonoscopy consent to baseline assessments and specimen/data collection (blood, urine, stool, saliva, breath and colon mucosal biopsies) at the time of colonoscopy. Following an 8-week stabilisation period to re-establish normal gut microbiota post colonoscopy, study personnel randomise participants to either a high fibre supplement (resistant starch, $\mathrm{n}=30$ ) or placebo (digestible starch, $\mathrm{n}=30$ ) condition, repeating stool sample collection. During the 28-day supplement trial, each participant consumes their usual diet plus their supplement under direct observation. On day 29, participants undergo a flexible sigmoidoscopy to obtain mucosal biopsy samples to measure the effect of the supplement on inflammatory and proliferative biomarkers of cancer risk, with follow-up assessments and data/specimen collection similar to baseline. Secondary outcome measures include the impact of a high fibre supplement on the oral and colonic microbiome and biofluid metabolome.

Ethics and dissemination Approvals were obtained from the Alaska Area and University of Pittsburgh Institutional Review Boards and Alaska Native Tribal Health Consortium and Southcentral Foundation research review bodies. A data safety monitoring board, material transfer agreements and weekly study team meetings provide regular oversight throughout the study. Study findings will first be shared with AN tribal leaders, health administrators, providers and community members. Peer-reviewed journal articles and conference presentations will be forthcoming once approved by tribal review bodies.

Trial registration number NCT03028831.
Strengths and limitations of this study

- Compliance of participants in the study has been excellent, due in part to the directly observed consumption of the supplementation or visualisation using real-time video via smartphone technologies.

- We have a strong and dedicated study team and a participant population very interested in helping to prevent colorectal cancer (CRC).

- While the population has high incidence of CRC and many patients are scheduled for screening colonoscopy, multiple exclusion criteria appropriate for this study limit the numbers eligible, especially in the older age range.

- In addition to slow and limited enrolment, the study was interrupted for 3 months during the COVID-19 pandemic, resulting in enrolment falling behind schedule.

\section{INTRODUCTION}

Diet is an environmental factor shown to impact colorectal cancer (CRC) risk by its effects on the colonic epithelium. ${ }^{1}$ Dietary fibre decreases CRC risk, whereas animal fat and red meat increase CRC risk. ${ }^{2}$ The human gut contains trillions of bacterial cells composing the gut microbiota. ${ }^{3}$ Dependent on food residues for survival and metabolism, gut microbiota play an important role in human health and metabolic homeostasis through their production of metabolites. Diets high in fat stimulate bile acid production in the liver. While the vast majority of bile acids are reabsorbed at the terminal ileum, approximately $5 \%$ are excreted into the colon. In the colon, primary bile acids are converted by gut bacteria into secondary bile acids that exhibit neoplastic properties. Conversely, fermentation of fibre by gut bacteria produces metabolites. Short-chain fatty acids (SCFA), including acetate, propionate and butyrate, not only provide food for 
the colonic epithelium but also exert regulatory effects on colonic mucosal inflammation and proliferation. ${ }^{45}$

Age-adjusted annual CRC incidence among Alaska Native (AN) people is the highest reported worldwide, ${ }^{6}$ with an annual Alaska-wide rate of 89/100 000. ${ }^{7}$ This rate is nearly double that of African Americans (45/100 000 per year), who have the second highest incidence in the USA and stands in stark contrast to other populations, such as rural Africans (RAs), with less than 5/100 000 new cases annually. ${ }^{8}$ Remarkably, the high CRC incidence reported in AN people is relatively recent, as chronic diseases were rarely documented a mere 65 years ago. ${ }^{9}$ While cancer case reporting as well as healthcare and life expectancy have improved over this time period, ${ }^{9-11}$ the very high incidence of CRC signals an urgent need for research to identify probable causes and propose important solutions to reduce AN CRC rates and risks.

AN people have faced and adapted to vast cultural and lifestyle changes, not the least of which have been dramatic dietary changes. ${ }^{12-14}$ These changes have resulted in a transition away from traditional, nutrientdense subsistence foods to commercially prepared, highenergy foods and beverages low in nutritional value..$^{15-19}$ Importantly, fibre, which plays a major role in establishing and maintaining colon health, is a key ingredient missing in what is described as a 'westernised' diet. Recent dietary studies conducted with AN people demonstrating a very low intake of approximately $13 \mathrm{~g}$ fibre daily ${ }^{15} 171920$ led us to collect self-reported diet intake and analyse stool contents in two groups of AN people living in two regions of the state: urban south central Alaska and the rural remote Arctic Slope region. ${ }^{21}$

In that study, participants scheduled for routine screening colonoscopy collected morning faecal and urine sample for metabolite analysis, then underwent colonoscopy. ${ }^{21}$ Results were compared with RA subjects enrolled separately, demonstrating clear differences between the two populations. While polyps were detected among $50 \%$ of AN participants, none were detected in the RA participants. The metabolic profiles of both urine and faecal samples were also significantly different. Levels of the faecal SCFA acetate, propionate and butyrate were significantly lower in the AN than the RA samples, while levels of the faecal primary bile acids (cholic acid and chenodeoxycholic acid) and secondary bile acid deoxycholic acid were significantly greater in AN, compared with RA samples.

Based on our knowledge of fibre and operating under the assumption that diet intake determines colon microbiota composition, the Alaska Native Medical Center (ANMC) and the University of Pittsburgh (U Pitt) designed the Alaska Fiber Intervention Research Study randomised clinical trial to demonstrate the effects of supplemented diet achieving at least $50 \mathrm{~g}$ /day of dietary fibre on CRC risk in AN study volunteers. We describe study methods to achieve our following study aims: (1) determine if high fibre intake can induce clinically meaningful suppression of proliferative and inflammatory colonic biomarkers of cancer risk (primary outcome) and (2) investigate our hypothesis that the high risk of colon cancer in AN people is a consequence of insufficient fibre intake leading to butyrate deficiency.

\section{METHODS AND ANALYSIS \\ Study design overview}

Using a parallel fibre and control group design, randomised by minimisation, ${ }^{22}{ }^{23}$ we are measuring the effects of a 4-week fibre (resistant starch) supplement compared with a 4-week placebo (digestible starch) supplement to participants' normal diet on proliferative changes in colonic mucosal cells. We anticipate the fibre supplement group will demonstrate significantly reduced proliferative biomarkers in biopsies obtained after the 4-week resistant starch supplement than the control group (primary endpoint). We will additionally measure and compare colonic microbiota composition, activity and the metabolome between groups to investigate the mechanisms responsible for differences in biomarkers.

The study is tied to usual care. Patients undergoing a routine screening colonoscopy, who are eligible and willing to participate, receive baseline assessments and specimen/data collection at the time of colonoscopy (see figure 1). This enrolment process is followed by an 8-week stabilisation period intended to re-establish normal gut microbiota post colonoscopy. At the end of stabilisation, study personnel randomise participants to either the fibre or placebo condition. During the next 28 days, each participant consumes their usual diet plus the supplement (fibre or placebo). After 4 weeks of supplement, participants voluntarily undergo a flexible sigmoidoscopy (flex sig) with specimen/data collection to test outcomes.

\section{Study setting}

ANMC is the sole tertiary healthcare facility serving more than $170000 \mathrm{AN}$ and American Indian people living in Alaska; the centre of the Alaska Tribal Health System (ATHS) is owned and operated by AN people. ${ }^{24}$ As a healthcare system, AN healthcare is the ATHS's primary focus, in contrast to academic and research institutions which focus on expanding scientific knowledge. ${ }^{25}$ As this study demonstrates, these foci can overlap, producing the opportunity to collaborate on separate, but aligned goals to expand scientific knowledge while simultaneously improving public health.

\section{Patient and public involvement}

Patients were not directly involved in protocol development. AN providers and staff contributed to study development on behalf of patients and tribal research review staff and board members evaluated, edited and approved the protocol and all study materials. Clinic personnel participated in developing procedures that would minimise their time and effort on study.

\section{Participants and recruitment}

We advertise the study using multiple venues, including tabling at large AN public gatherings, health fairs and 


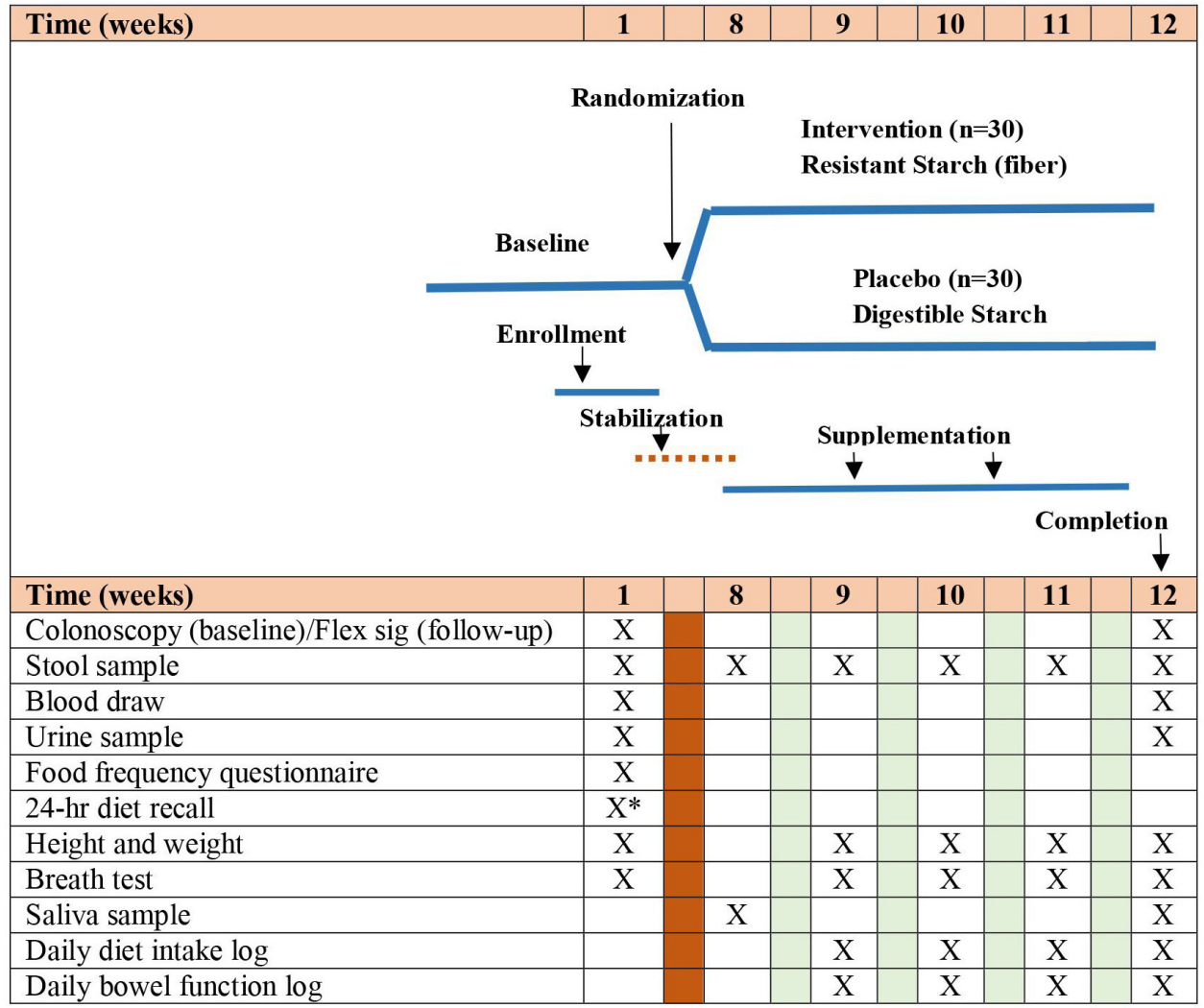

Figure 1 Study design and timeline. *Twenty-four hour diet recall interviews are generally completed after the baseline colonoscopy and prior to randomisation and supplementation.

tribal events. We have study banners in clinic waiting areas, flyers posted in primary care clinics, Facebook announcements incorporating YouTube videos and AN digital stories and advertisements in local newspapers. Certified medical assistants (CMAs) and reception staff assist by introducing patients to the study and introducing them to study staff with a 'warm handoff'.

Due to the high incidence of CRC at a relatively young age among AN people, CRC screening beginning at age 40 years is standard of care; colonoscopy is the recommended screening test at ANMC. AN patients scheduling a screening colonoscopy through the CRC clinic are invited to participate in the study if they meet eligibility criteria. A trained study nurse with access to CRC clinic schedules prescreens electronic medical records of patients scheduled for a preoperative (preop) visit for inclusion/exclusion criteria 1-3 days prior to their scheduled preop appointment. Specific inclusion and exclusion criteria are provided in table 1 .

Following prescreening, the study nurse provides the clinic CMA with information on potential participants. Checking in for their appointment, CRC clinic staff direct these patients to complete the clinical paperwork, asking them to also review the study flyer. Clinic staff then direct interested patients to study staff.

\section{Enrolment phase}

We opened enrolment on 27 November 2017, with the goal to enrol 60 participants (30 participants in each study arm) who will complete the study. We determined statistical power based on mucosal proliferation (measured by Ki-67 protein), the primary endpoint, using changes observed in a 2-week fibre supplementation study with African Americans. ${ }^{26}$ While a $20 \%$ difference between fibre and placebo implies clinical significance, we have at least $80 \%$ power to detect a reduction of at least $8 \%$ in the fibre arm from the placebo arm, using a two-sided hypothesis test at $\alpha=0.05$. We will also have more than adequate power for secondary endpoints described in table $1 .{ }^{26} \mathrm{We}$ anticipate enrolling up to 100 participants to achieve 60 study completions, with attrition due to postcolonoscopy ineligibility, participant scheduling conflicts, losses to follow-up and supplement intolerance. The enrolment phase consists of baseline data and procedures collected the day before, day of, during and after colonoscopy before randomisation (see figure 1).

\section{Day before colonoscopy}

Participants are usually prepped for colonoscopy the evening before colonoscopy. A study team member observes intake of the polyethylene glycol (PEG) solution that is free of sulfates, which are harmful to colon microbiota. No pre-prep instructions are required. Prior to prep ingestion, study personnel obtain participant height (via study stadiometer) and weight (via study scale) then calculate baseline body mass index (BMI) per the standard formula (weight in kilograms divided by height in metres, squared). Study personnel assess colonic fermentation 
Table 1 Inclusion/exclusion criteria for the Alaska FIRST clinical trial

\begin{tabular}{ll}
\hline Category & Description \\
\hline Inclusion & Alaska Native race (eligible for Indian Health \\
& Service Care) \\
& Age $40-75$ years \\
& BMI $18-40 \mathrm{~kg} / \mathrm{m}^{2}$ \\
& Living within the south central (Anchorage, \\
& Alaska) area \\
& Scheduled at ANMC for a routine colonoscopy \\
Exclusion & Family history of familial adenomatous \\
& polyposis, Lynch syndrome or hereditary non- \\
polyposis CRC & Previously diagnosed with inflammatory bowel \\
disease or colon cancer & Personal history of kidney or liver disease or \\
bleeding disorder & Previous gastrointestinal surgery resulting in \\
disturbed gut function due to loss of bowel or \\
altered anatomy \\
Any form of chronic gastrointestinal disease \\
resulting in disturbed gut function, diarrhoea or \\
malabsorption were excluded from the study \\
Antibiotic treatment within the past 12 weeks \\
Current and regular treatment with steroids \\
Prescribed diabetes medication
\end{tabular}

ANMC, Alaska Native Medical Center; BMI, body mass index; CRC, colorectal cancer; FIRST, Fiber Intervention Research Study.

measuring hydrogen $\left(\mathrm{H}_{2}\right)$ and methane $\left(\mathrm{CH}_{4}\right)$ production via a breath sample, which they analyse on collection using a commercial analyzer (Quintron BreathTracker SC). Participants are also asked to provide a urine sample, from which $33 \mathrm{~mL}$ is aliquotted and stored in $-80^{\circ} \mathrm{C}$ for later analysis of microbial metabolites. Participants then consume 2.0-2.5 L of the sulfate-free PEG solution in $30-60 \mathrm{~min}$.

During the next few hours, study personnel collect and aliquot the first solid stool passed (which will be analysed for faecal microbes and metabolites), placing it in $-80^{\circ} \mathrm{C}$ and collect remaining participant evacuate for the next 3 hours. Once the 3-hour evacuate is collected (which will be analysed for total colonic microbiota and their metabolites), study staff weigh, homogenise, aliquot and freeze specimens at $-80^{\circ} \mathrm{C}$; the 3-hour evacuate collection period provides sufficient time to obtain a food frequency questionnaire (FFQ) developed and validated for use with AN study participants ${ }^{27}$ and a baseline bowel function assessment. The FFQ is intended to provide a list of foods and approximate proportions consumed within the past year. In addition to commercial foods, the FFQ contains common AN subsistence foods, including Alaska fish and seafood, wild game and birds, bird eggs and tundra berries and greens traditionally prepared and preserved. The standardised bowel function assessment ${ }^{28}$ (assessing usual bowel habits, frequency, consistency) has been employed in prior U Pitt studies. ${ }^{8}{ }^{26}$ On completion of the prep and precolonoscopy procedures, participants have nothing more by mouth and report to the surgery suite at their designated time with an escort.

\section{Day of colonoscopy prior to procedure}

The ANMC surgeon/endoscopist performs colonoscopy under conscious sedation per standard clinical procedure. Clinical staff placing the intravenous line draw off $30 \mathrm{cc}$ of blood for study purposes prior to starting the fluid drip. Study staff transfer blood collected for the study to the appropriate tubes for ANMC lab analyses and future U Pitt analyses. Baseline study analyses performed at the ANMC lab include: fasting blood count, complete metabolic profile, lipoproteins, triglycerides, folate and vitamins B-12 and D to assess nutritional status. Study staff additionally prepare and aliquot whole blood and serum samples, which are placed in $-80^{\circ} \mathrm{C}$ until shipped to $\mathrm{U}$ Pitt for additional analyses (see table 1).

\section{Intraoperative}

In addition to any clinical biopsies necessary, study biopsies are taken from normal-appearing mucosa at the cecum, mid-traverse and sigmoid colon $(25 \mathrm{~cm}$ from the anal verge). Study biopsy material will be used to measure colon cancer biomarkers and mucosa-attached microbes. One biopsy at each region is flash frozen in liquid nitrogen and placed in a $-80^{\circ} \mathrm{C}$ freezer. A second biopsy from each region is placed in RNAlater solution, flash frozen in liquid nitrogen, refrigerated overnight and the RNAlater solution pipetted off the next day before being frozen at $-80^{\circ} \mathrm{C}$. A third biopsy at each region is preserved in formalin and stored at room temperature.

\section{Post colonoscopy prior to randomisation}

The study nurse reviews the participant's colonoscopy results to determine continued study eligibility. If colonoscopy findings do not include any items listed in the exclusion criteria, participants remain eligible for study participation. At this point, participants enter the stabilisation phase. This phase overlaps with the enrolment phase until study staff collect all enrolment materials, which include two 24-hour diet recalls, 1 week day and 1weekend day, using the Nutrition Data System for Research (NDSR, Nutrition Coordinating Center, University of Minnesota, Minneapolis, Minnesota; versions 2017 through 2020). ${ }^{29}$ Study staff conducting 24-hour recalls are trained in NDSR procedures, having completed 10 practice collections and a final mock collection interview with a NDSR-prepared study staff member. On enrolment phase completion, we provide participants a $\$ 100$ enrolment gift card in appreciation for their time and effort.

\section{Stabilisation phase}

During the stabilisation phase, participants resume their usual diet. An 8-week stabilisation period is intended to re-establish resident microbiota eliminated with the 
colonoscopy prep. ${ }^{263031}$ During this period, study activities are limited to participant randomisation and establishing a supplementation phase start date, both occurring at the end of the stabilisation phase.

We use minimisation as the method of randomisation. Minimisation is better than simple randomisation for balancing prognostic factors that could bias study outcomes between arms. ${ }^{22} 23$ In this study, minimisation assigns participants to fibre versus control groups to maintain the balance of the following factors, listed in order of priority: prior polypectomy, sex, BMI $\left(<30 \mathrm{~kg} / \mathrm{m}^{2}\right.$ versus $\left.\geq 30 \mathrm{~kg} / \mathrm{m}^{2}\right)$ and fish consumption $(<4$ servings per week versus $\geq 4$ servings). Minimisation is implemented via an $\mathrm{R}$ script with an e-mail front end written in Python 2.6 running at $\mathrm{U}$ Pitt.

On assignment, Alaska Native Tribal Health Consortium (ANTHC) Study staff are provided a randomly numbered code to assign to each new participant. These codes match coded labels on the supplement packets previously mailed to the ANTHC Study team and are the only information provided on the packets. Only designated U Pitt Study team members have access to the identity of the supplement and only ANTHC Study staff can link Study IDs to participant identifiers, so the double blind and participant confidentiality are maintained. Should the need arise to identify which product an individual is assigned (eg, complaints of supplement intolerance), a two-person process is in place.

\section{Supplementation phase}

The supplementation phase begins with supplement initiation. Study personnel contact participants to set up their direct observed supplement intake for 28 consecutive days. Study staff work closely with participants to time the supplement so that the date of follow-up flex sig performed on the 29th day is convenient for the participant, available and scheduled in advance. Prior to initiating supplementation, study staff collect presupplement weight as well as stool and saliva samples. Both samples are stored frozen at $-80^{\circ} \mathrm{C}$ until shipped to U Pitt for analysis. We plan to analyse saliva samples collected using an OMNIgene oral kit (DNA Genotek, Inc.) to compare oral and colon microbiomes pre and post supplementation.

Study supplement packets for both fibre and placebo groups are prepared by Ingredion (Bridgewater, New Jersey) and shipped to U Pitt. For the fibre group, supplement is given as a daily dose of $70 \mathrm{~g}$ high-amylose maize starch, containing $42 \mathrm{~g}$ of resistant starch. Supplements are analysed by the Englyst method to confirm the precise amount of resistant starch given. ${ }^{32}$ Data from previous AN studies suggest that the usual AN diet contains approximately $13 \mathrm{~g}$ of fibre per day. ${ }^{151719}$ Thus, total fibre intake would be about $55 \mathrm{~g}$ per day, a quantity sufficient to suppress colon cancer biomarkers (see table 2 measured outcomes) after 2 weeks in previous studies. ${ }^{26} 3334$

While participants in the placebo group also continue their usual diet, supplement packets for this group (also prepared by Ingredion) contain $35 \mathrm{~g}$ of fully digestible starch (ie, corn starch), weighed, analysed, prepared and presented in the same way. Fibre and placebo supplements are equal in calories, packaged identically in daily dose packets and appear and taste similar, thus maintaining the double blind. Due to the substantial increase of fibre from 13 to $55 \mathrm{~g} /$ day, previous tolerance tests with $\mathrm{AN}$ volunteers prior to this study were performed and demonstrated the supplement was well tolerated as a single or divided dose mixed into $300 \mathrm{~mL}$ water taken for a period of a week.

Ingredion sends complete batches of single-dose packets of each supplement type to U Pitt, where U Pitt Study staff apply assignment code labels corresponding to the codes generated by the electronic minimisation programme, plus a daily sequence number (day 1-day 35 ). Batches of 35 labelled packets are shipped in advance to the ANTHC Study team. Once participants are randomised and assigned a supplement code, ANTHC Study staff locate the packet batch. An additional week of packets are available should a flex sig procedure be unexpectedly delayed.

Each participant is provided a shaker bottle and instructions (with in-person demonstration, as needed) for mixing the supplement packet with $300 \mathrm{~mL}$ water (or other preferred liquid). Study staff work closely with each participant to plan 28 consecutive days where each participant is directly observed mixing and drinking the supplement, which can be done in-person or via FaceTime or Google Duo and includes weekends and holidays. The first supplement is done in the presence of study staff for quality assurance, reserving a $5 \mathrm{mg}$ sample to validate content and requesting participants assume usual diet and take the supplement about the same time each day.

Study staff also provide instruction on completing a daily food $\log$ and daily bowel function form and provide collection supplies for the first stool the next week. At the completion of each week, study staff collect all completed daily food logs, bowel function forms and an end-of-week stool sample. Participants are reweighed and given their weekly stool sample collection kit and the next week's supply of supplement. Participant weight and supplement tolerance are thus monitored throughout the supplementation period. Any participant with a $2 \mathrm{~kg}$ change in weight or gastrointestinal complaints during the supplementation phase is referred to the study dietician for evaluation. On completion of all weekly supplement intake, assessment forms, weight and sample collections, each participant receives a $\$ 75$ gift card.

On entering the fourth week of supplementation, study staff prepare for the study flex sig procedure. Measurements (except height) and specimens collected pre colonoscopy are repeated. Participants revisit the CRC clinic preoperatively and prior to starting the sulfate-free PEG prep solution, study staff collect a saliva sample. The rest of the flex sig prep is conducted similarly to the baseline colonoscopy.

Previous studies show changes in the sigmoid mucosa reflect changes in the proximal colon. ${ }^{26}{ }^{35} \mathrm{~A}$ flex sig is thus sufficient for postsupplementation biopsies. Three 


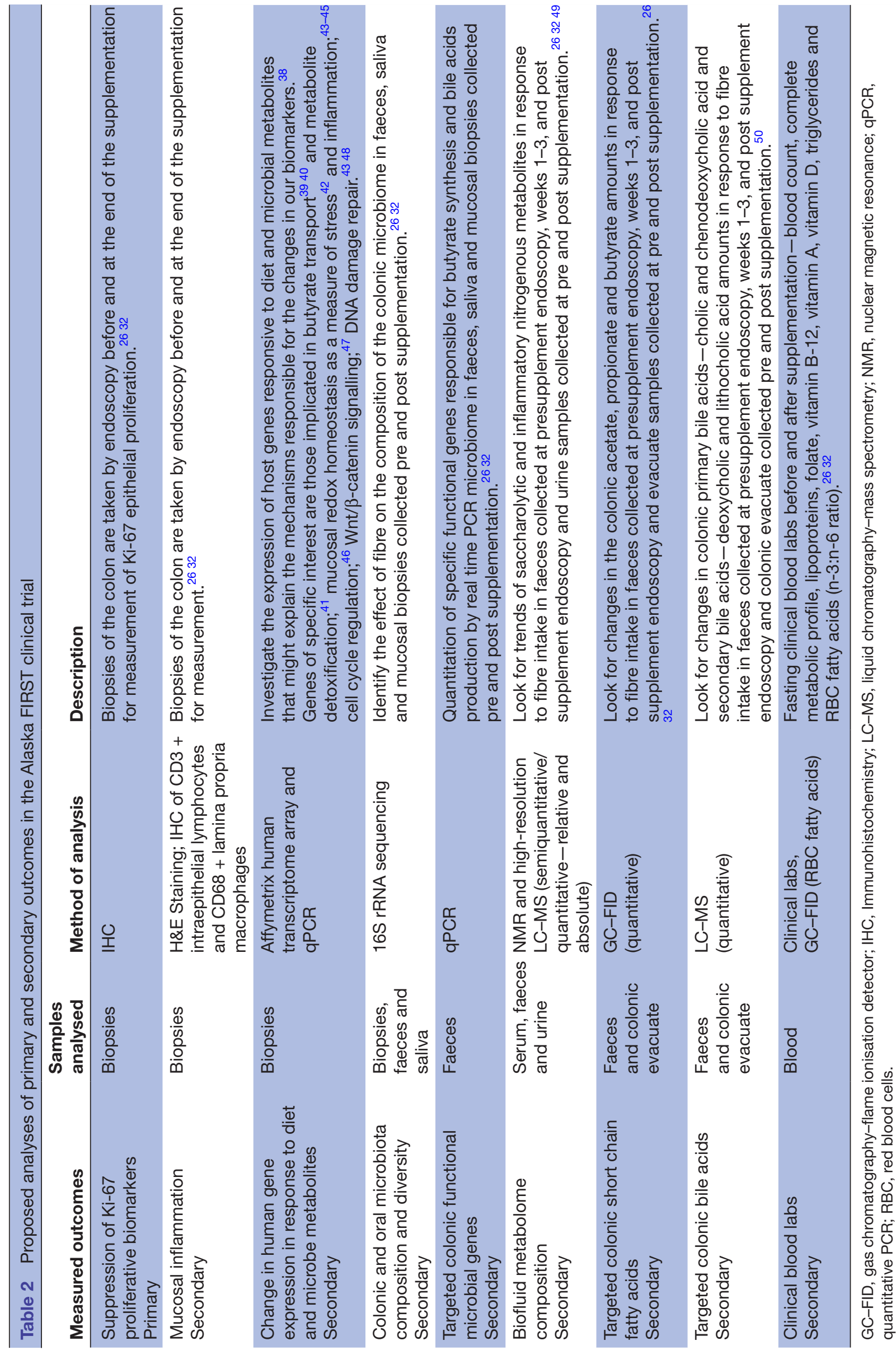


biopsies from normal appearing mucosa in two regions of the colon, the sigmoid ( $25 \mathrm{~cm}$ from the anal verge) and the splenic flexure (or the furthest region easily accessed), are obtained by an ANMC surgeon/endoscopist. On procedure completion, participants receive a $\$ 300$ gift card in appreciation for their participation.

\section{Data entry/specimen storage and transfer to U Pitt}

The large number of procedures performed and specimens/data collected throughout this trial require detailed documentation, accurate labelling and strict attention to protocol. Working with ANMC providers and staff in clinics, preop and endoscopy suites and laboratory and pathology departments, ANMC and U Pitt Study teams created more than 90 standard operating procedures and clinical report forms (CRFs) contained in the study manual of operations. Because procedure data and biospecimen collection do not all occur in a set location with reliable access to Wi-Fi or the internet, much of the documentation is initially charted on paper forms and maintained in participant folders. All data and specimens collected are associated with only an anonymous study identification number and accessible only to the ANTHC Study team.

Study team members enter all CRF data into Research Electronic Data Capture (REDCap) databases housed within protected servers at ANTHC and U Pitt, each containing different data elements. REDCap is a secure, web-based, data capture application designed for research studies. ${ }^{36}$ The U Pitt database, made accessible to the ANTHC Study team, houses data relevant to biospecimens (blood, urine, stool and saliva aliquots and biopsy samples), which study staff batch and ship to U Pitt for analysis throughout the study. All but the formalin preserved tissue samples are frozen at $-80^{\circ} \mathrm{C}$ and housed first in ANTHC Study freezers then shipped overnight on dry ice by FedEx to U Pitt. A second REDCap database created by the ANTHC Study team captures CRF information, including laboratory and anthropometry data, daily food intake and bowel function information and breath and saliva sample collection data. A third database (also housed at ANTHC), directly captures FFQ data collected during the baseline colonoscopy prep period. Institutional database administrators set individual data access to specific data and the level of access (eg, data entry, data download, view only), including access for external collaborators.

\section{Proposed analyses}

Study analyses are designed to test the hypothesis that the risk of CRC in AN people is primarily a consequence of fibre intake less than the recommended $30 \mathrm{~g}$ /day. The primary outcome measured in our study is colonic epithelial proliferation rate measured by Ki67 staining of proliferative cells to determine whether a high fibre diet can induce clinically meaningful (ie, $>20 \%$ the difference distinguishing high from low risk populations, termed 'responders') suppression of this proliferative cancer risk biomarker. ${ }^{87}$ In addition, secondary outcomes include inflammatory markers of the gut mucosa and the impact of a high fibre diet on expression of host genes responsive to diet and microbial metabolites, clinical blood labs, faecal and oral microbiome and faecal, blood and urine metabolome. We will explore the impact of the gut and oral microbiome and faecal, urine and blood metabolome on host biomarkers and host-microbe cometabolism. We will assess postsupplement changes in markers by generalised linear models. The nature of the data will determine whether these models are linear, logistic, Poisson or require further transformation. Table 2 contains more detailed information regarding the measured endpoints.

\section{ETHICS AND DISSEMINATION}

This ongoing 5-year study is funded by the National Institutes of Health through a National Cancer Institute grant (CA204403) awarded to the U Pitt with a subaward to the ANTHC.

\section{Permissions and approvals}

The U Pitt and Alaska Area institutional review boards (IRBs) reviewed and approved this study. Boards of directors for ANTHC and Southcentral Foundation (SCF) provided tribal review and approval. ANTHC and SCF are tribal health organisations (THOs) operating the ANMC located in Anchorage, Alaska. While IRBs focus on the protection of human participants in research, THOs focus attention on protecting AN communities and culture. Tribal approval is required for all health research proposals with AN people.

\section{Study oversight}

All clinical trials supported by the National Cancer Institute (NCI) require monitoring. Monitoring should reflect the degree of risk involved in participation of the clinical trial. We established a data safety monitoring board (DSMB), fully executed institutional material transfer agreements with all collaborators and created an internal investigator team, providing these additional layers of study oversight distinct from the IRB and tribal review process.

The DSMB is comprised of three external experts in nutrition and gastrointestinal research, the study principal investigator, the ANTHC project director and the NCI programme officer. Meeting annually, the DSMB reviews an annual report of research activities and addresses analyses performed, preliminary outcome data, adverse side effects or toxicity caused by treatments, inclusion and exclusion criteria, enrolment procedures and modifications to any elements of the study.

Signed material transfer agreements between all collaborating institution clearly state the terms under which tribal data and specimens will be shared, maintained and destroyed and results reported. U Pitt and ANTHC investigators and study staff meet weekly (virtually or by teleconference) to discuss study progress and address 
challenges. Additional attendees are present on request (eg, U Pitt biostatistician, data managers).

\section{Results dissemination}

Tribal review of research findings is the first step in results dissemination, per ANTHC research policy. We will provide early reports and presentations of the findings to ANTHC and SCF Board members, health administrators and healthcare providers. As these study findings most directly affect AN people, dissemination plans include mass media announcements and messaging through ANTHC's Marketing Department. Because this study will demonstrate important findings relevant to other populations having transitioned to a westernised diet, we will inform the scientific community through professional presentations and peer-reviewed journal articles that have received tribal review and approval prior to submission.

\section{Author affiliations}

${ }^{1}$ Research Services, Alaska Native Tribal Health Consortium, Anchorage, Alaska, USA ${ }^{2}$ Department of Medicine, University of Pittsburgh, Pittsburgh, Pennsylvania, USA ${ }^{3}$ Hillman Cancer Center Biostatistics Facility, University of Pittsburgh, Pittsburgh, Pennsylvania, USA

${ }^{4}$ Australian National Phenome Center, Murdoch University, Perth, Western Australia, Australia

${ }^{5}$ Department of Metabolism, Digestion and Reproduction, Imperial College London, London, UK

${ }^{6}$ Department of Surgery and Cancer, Imperial College London, London, UK ${ }^{7}$ Alaska Native Epidemiology Center, Alaska Native Tribal Health Consortium, Anchorage, Alaska, USA

${ }^{8}$ Wellness and Prevention, Alaska Native Tribal Health Consortium, Anchorage, Alaska, USA

Acknowledgements The authors wish to express their appreciation to Alaska Native Tribal Health Consortium and Southcentral Foundation leadership for their support to the study and to members of the research review committees for their thorough review and approval of this manuscript. The authors deeply appreciate all providers and staff members in the Alaska Native Medical Center clinics, operating rooms and laboratory/histology departments who provided input and support for this protocol in so many ways. The authors wish to thank their Alaska Native participants for participating in this study. This study would not be possible without the combined efforts of authors' study team and all those mentioned above.

Contributors Primary lead author was KRK and secondary lead was AW. Authors KRK and AW outlined and wrote the original draft of the manuscript. DPN designed randomisation by minimisation and contributed to writing this section. JKN contributed to statistical analysis. JVL and JK contributed to the background and design overview. FRL, CAF and ZTM contributed edits to study operations and specimen acquisition, processing, storage and shipping. PI and DLL reviewed and edited the sections pertaining to data management and security. TKT oversees the study in Alaska as the site PI and SJDO is the study PI located at University of Pittsburgh. All authors were involved in the study design and implementation and reviewed and contributed edits to the drafts of the paper.

Funding This work was supported by the National Institutes of Health through the National Cancer Institute grant number CA204403.

Competing interests JK reports grants from H2020 - ITN grant, grants from NIHR-i4i grant, grants from CRUK fellowship and grants from J+J Educational grant, personal fees from Verb Robotics/Ethicon and Medtronic, outside the submitted work, and others from Cerulean Health, OneWelbeck and LNC Therapeutics. No other authors have a competing interests to disclose.

Patient and public involvement Patients and/or the public were not involved in the design, or conduct, or reporting, or dissemination plans of this research.

Patient consent for publication Not required.

Provenance and peer review Not commissioned; externally peer reviewed.
Open access This is an open access article distributed in accordance with the Creative Commons Attribution Non Commercial (CC BY-NC 4.0) license, which permits others to distribute, remix, adapt, build upon this work non-commercially, and license their derivative works on different terms, provided the original work is properly cited, appropriate credit is given, any changes made indicated, and the use is non-commercial. See: http://creativecommons.org/licenses/by-nc/4.0/.

ORCID iDs

Kathryn R Koller http://orcid.org/0000-0002-4066-0930

Annette Wilson http://orcid.org/0000-0002-7426-1786

\section{REFERENCES}

1 Doll R, Peto R. The causes of cancer: quantitative estimates of avoidable risks of cancer in the United States today. J Natl Cancer Inst 1981;66:1192-308.

2 World Cancer Research Fund, American Institute for Cancer Research. Food, nutrition, physical activity, and the prevention of colorectal cancer (continuous update project report), 2011. Available: http://www.wcrf.org/sites/default/files/Colorectal-Cancer-2011Report.pdf

3 Sender R, Fuchs S, Milo R. Revised estimates for the number of human and bacteria cells in the body. PLoS Biol 2016;14:e1002533.

4 Topping DL, Clifton PM. Short-chain fatty acids and human colonic function: roles of resistant starch and nonstarch polysaccharides. Physiol Rev 2001;81:1031-64.

5 O'Keefe SJD. Diet, microorganisms and their metabolites, and colon cancer. Nat Rev Gastroenterol Hepatol 2016;13:691-706.

6 Siegel RL, Miller KD, Goding Sauer A, et al. Colorectal cancer statistics, 2020. CA Cancer J Clin 2020;70:145-64.

7 North American Association of Central Center Registries (NAACCR). Fast STATs 2012-2016 cancer incidence data. Available: https://www. naaccr.org/interactive-data-on-line/

8 O'Keefe SJD, Chung D, Mahmoud N, et al. Why do African Americans get more colon cancer than native Africans? J Nutr 2007;137:175S-82.

9 Parran T, Ciocco A, Crabtree JA. Alaska's health: a survey report to the United States Department of the Interior. Pittsburgh, PA: Graduate School of Public Health, University of Pittsburgh, 1954.

10 Day GE, Lanier AP. Alaska Native mortality, 1979-1998. Public Health Rep 2003;118:518-30.

11 Alaska Native Epidemiology Center. Alaska Native health data: life expectancy, 2020. Available: http://anthctoday.org/epicenter/

12 Murphy NJ, Schraer CD, Theile MC, et al. Hypertension in Alaska Natives: association with overweight, glucose intolerance, diet and mechanized activity. Ethn Health 1997;2:267-75.

13 Nobmann ED, Byers T, Lanier AP, et al. The diet of Alaska Native adults: 1987-1988. Am J Clin Nutr 1992;55:1024-32.

14 Schraer CD. Diabetes among the Alaska Natives - The emergence of a chronic disease with changing life-styles. In: Joe J, ed. Diabetes as a disease of civilization: the impact of culture change on Indigenous peoples. Berlin, New York: Mouton de Gruyter, 1994: 169-93.

15 Bersamin A, Luick BR, Ruppert E, et al. Diet quality among Yup'ik Eskimos living in rural communities is low: the center for Alaska Native health research pilot study. J Am Diet Assoc 2006;106:1055-63.

16 Bersamin A, Zidenberg-Cherr S, Stern JS, et al. Nutrient intakes are associated with adherence to a traditional diet among Yup'ik Eskimos living in remote Alaska Native communities: the CANHR study. Int J Circumpolar Health 2007;66:62-70.

17 Johnson JS, Nobmann ED, Asay E, et al. Dietary intake of Alaska Native people in two regions and implications for health: the Alaska Native Dietary and Subsistence Food Assessment project. Int J Circumpolar Health 2009;68:109-22.

18 Bersamin A, Luick BR, King IB, et al. Westernizing diets influence fat intake, red blood cell fatty acid composition, and health in remote Alaskan Native communities in the center for Alaska Native health study. J Am Diet Assoc 2008;108:266-73.

19 Johnson JS, Nobmann ED, Asay E. Factors related to fruit, vegetable and traditional food consumption which may affect health among Alaska Native people in western Alaska. Int J Circumpolar Health 2012;71:17345

20 Redwood DG, Ferucci ED, Schumacher MC, et al. Traditional foods and physical activity patterns and associations with cultural factors in a diverse Alaska Native population. Int J Circumpolar Health 2008;67:335-48.

21 Ocvirk S, Wilson AS, Posma JM, et al. A prospective cohort analysis of gut microbial co-metabolism in Alaska Native and rural African 
people at high and low risk of colorectal cancer. Am J Clin Nutr 2020;111:406-19.

22 Treasure T, MacRae KD. Minimisation: the platinum standard for trials?. Randomisation doesn't guarantee similarity of groups; minimisation does. BMJ 1998;317:362-3.

23 Pocock SJ, Simon R. Sequential treatment assignment with balancing for prognostic factors in the controlled clinical trial. Biometrics 1975;31:103-15.

24 Sherry P. Health care delivery for Alaska Natives: a brief overview. Int J Circumpolar Health 2004;63:54-62.

25 Alaska Native Tribal Health Consortium. ANTHC overview, 2020.

26 O'Keefe SJD, Li JV, Lahti L, et al. Fat, fibre and cancer risk in African Americans and rural Africans. Nat Commun 2015;6:6342.

27 Johnson JS, Nobmann ED, Asay E, et al. Developing a validated Alaska Native food frequency questionnaire for western Alaska, 2002-2006. Int J Circumpolar Health 2009;68:99-108.

28 Talley NJ, Phillips SF, Wiltgen CM, et al. Assessment of functional gastrointestinal disease: the bowel disease questionnaire. Mayo Clin Proc 1990;65:1456-79.

29 University of Minnesota Nutrition Coordinating Center (NCC). NDSR software, 2020.

30 Mai V, Greenwald B, Morris JG, et al. Effect of bowel preparation and colonoscopy on post-procedure intestinal microbiota composition. Gut 2006;55:1822-3.

31 O'Brien CL, Allison GE, Grimpen F, et al. Impact of colonoscopy bowel preparation on intestinal microbiota. PLoS One 2013;8:e62815

32 Englyst HN, Kingman SM, Hudson GJ, et al. Measurement of resistant starch in vitro and in vivo. Br J Nutr 1996;75:749-55.

33 Burkitt DP. Epidemiology of cancer of the colon and rectum. Cancer 1971;28:3-13.

34 Bultman SJ. Molecular pathways: gene-environment interactions regulating dietary fiber induction of proliferation and apoptosis via butyrate for cancer prevention. Clin Cancer Res 2014;20:799-803.

$35 \mathrm{Ou}$ J, Carbonero F, Zoetendal EG, et al. Diet, microbiota, and microbial metabolites in colon cancer risk in rural Africans and African Americans. Am J Clin Nutr 2013;98:111-20.

36 Vanderbilt University. Research Electronic Data Capture (REDCap) About, 2004. Available: https://projectredcap.org/about/

37 Wood CE, Hukkanen RR, Sura R, et al. Scientific and regulatory policy committee (SRPC) review: interpretation and use of cell proliferation data in cancer risk assessment. Toxicol Pathol 2015;43:760-75.
38 Turnbaugh PJ, Gordon Jl. An invitation to the marriage of metagenomics and metabolomics. Cell 2008;134:708-13.

39 Cuff M, Dyer J, Jones M, et al. The human colonic monocarboxylate transporter isoform 1: its potential importance to colonic tissue homeostasis. Gastroenterology 2005;128:676-86.

40 Thibault R, Blachier F, Darcy-Vrillon B, et al. Butyrate utilization by the colonic mucosa in inflammatory bowel diseases: a transport deficiency. Inflamm Bowel Dis 2010;16:684-95.

41 Sauer J, Richter KK, Pool-Zobel BL. Physiological concentrations of butyrate favorably modulate genes of oxidative and metabolic stress in primary human colon cells. J Nutr Biochem 2007; 18:736-45.

42 Hamer HM, Jonkers DMAE, Bast A, et al. Butyrate modulates oxidative stress in the colonic mucosa of healthy humans. Clin Nutr 2009;28:88-93.

43 Agoff SN, Brentnall TA, Crispin DA, et al. The role of cyclooxygenase 2 in ulcerative colitis-associated neoplasia. Am J Pathol 2000;157:737-45.

44 Reddy BS, Hirose Y, Cohen LA, et al. Preventive potential of wheat bran fractions against experimental colon carcinogenesis: implications for human colon cancer prevention. Cancer Res 2000;60:4792-7.

45 Popivanova BK, Kitamura K, Wu Y, et al. Blocking TNF-alpha in mice reduces colorectal carcinogenesis associated with chronic colitis. $J$ Clin Invest 2008;118:560-70.

46 Shi T, Mazumdar T, Devecchio J, et al. cDNA microarray gene expression profiling of hedgehog signaling pathway inhibition in human colon cancer cells. PLoS One 2010;5 doi:10.1371/journal. pone.0013054

47 Zhang Y, Li Q, Zhou D, et al. Genistein, a soya isoflavone, prevents azoxymethane-induced up-regulation of Wnt/ $\beta$-catenin signalling and reduces colon pre-neoplasia in rats. Br J Nutr 2013;109:33-42.

48 Conlon MA, Kerr CA, McSweeney CS, et al. Resistant starches protect against colonic DNA damage and alter microbiota and gene expression in rats fed a Western diet. J Nutr 2012;142:832-40.

49 Dona AC, Jiménez B, Schäfer $\mathrm{H}$, et al. Precision high-throughput proton NMR spectroscopy of human urine, serum, and plasma for large-scale metabolic phenotyping. Anal Chem 2014;86:9887-94.

50 Sarafian MH, Lewis MR, Pechlivanis A, et al. Bile acid profiling and quantification in biofluids using ultra-performance liquid chromatography tandem mass spectrometry. Anal Chem 2015;87:9662-70. 